\title{
SNP array-based copy number and genotype analyses for preimplantation genetic diagnosis of human unbalanced translocations
}

\author{
Chris MJ van Uum ${ }^{1}$, Servi JC Stevens ${ }^{\star, 1}$, Joseph CFM Dreesen ${ }^{1,2}$, Marion Drüsedau1, Hubert J Smeets ${ }^{1,2}$, \\ Bertien Hollanders-Crombach ${ }^{1}$, Christine EM de Die-Smulders ${ }^{1,2}$, Joep PM Geraedts ${ }^{1,2}$, John JM Engelen ${ }^{1,2}$ \\ and Edith Coonen ${ }^{1,3}$
}

Preimplantation genetic diagnosis (PGD) for chromosomal rearrangements (CR) is mainly based on fluorescence in situ hybridisation (FISH). Application of this technique is limited by the number of available fluorochromes, the extensive preclinical work-up and technical and interpretative artefacts. We aimed to develop a universal, off-the-shelf protocol for PGD by combining single-nucleotide polymorphism (SNP) array-derived copy number (CN) determination and genotyping for detection of unbalanced translocations in cleavage-stage embryos. A total of 36 cleavage-stage embryos that were diagnosed as unbalanced by initial PGD FISH analysis were dissociated $(n=146)$ and amplified by multiple displacement amplification (MDA). SNP CNs and genotypes were determined using SNP array. Epstein-Barr Virus-transformed cell lines with known CR were used for optimising the genomic smoothing (GS) length setting to increase signal to noise ratio. SNP CN analysis showed 23 embryos (64\%) that were unbalanced in all blastomeres for the chromosomes involved in the translocation, 5 embryos (14\%) that were normal or balanced in all blastomeres and 8 embryos $(22 \%)$ that were mosaic. SNP genotyping, based on analysis of informative SNP loci with opposing homozygous parental genotypes, confirmed partial monosomies associated with inheritance of unbalanced translocation in surplus embryos. We have developed a universal MDA-SNP array technique for chromosome CN analysis in single blastomeres. SNP genotyping could confirm partial monosomies. This combination of techniques showed improved diagnostic specificity compared with FISH and may provide more reliable PGD analysis associated with higher embryo transfer rate.

European Journal of Human Genetics (2012) 20, 938-944; doi:10.1038/ejhg.2012.27; published online 29 February 2012

Keywords: preimplantation genetic diagnosis; SNP array; chromosomal translocations; whole-genome amplification; genotyping; blastomeres

\section{INTRODUCTION}

Carriers of structural chromosomal rearrangements (CR), such as reciprocal or Robertsonian translocations are at increased risk of genetically abnormal offspring. They are also at higher risk for spontaneous miscarriage due to unbalanced CR in their offspring. ${ }^{1-2}$ Prenatal testing allows for the detection of unbalanced offspring followed by eventual termination of the pregnancy. Preimplantation genetic diagnosis (PGD) offers a valuable alternative to prenatal genetic testing. To date, the majority of PGD cycles is performed on single blastomeres from in vitro fertilisation-derived cleavage-stage embryos, using fluorescence in situ hybridisation (FISH) (for chromosomal or specific X-linked recessive disorders). ${ }^{3}$ FISH requires extensive preclinical work-up, as it is pivotal to develop a diagnostic test tailored to the individual couple's chromosomal aberration. The majority of couples have a unique reciprocal translocation, requiring a personalised combination of probes. Thus, pre-PGD selection and validation of informative FISH probes is essential. Furthermore, FISH analysis of blastomeres is laborious and cannot be automated. It requires experienced technicians and is subjective with respect to interpretation of weak or dubious signals. Interpretation of FISH analysis is further complicated by occasional blastomere fragmentation or multinucleated blastomeres. Finally, only a limited number of discriminative FISH fluorochromes are available. ${ }^{4-6}$

Single-cell whole-genome amplification with subsequent use of a genome-wide array technique may overcome most of the drawbacks described above. ${ }^{7-10}$ It greatly increases the number of markers that can be analysed simultaneously and omits the need for extensive prePGD work-up, allowing rapid initiation of a PGD cycle and high diagnostic laboratory throughput. Genome-wide techniques such as array-comparative genomic hybridisation (CGH) and singlenucleotide polymorphism (SNP) array have been applied on single embryonic cells mainly for the detection of aneusomies of whole chromosomes in preimplantation genetic screening (PGS). ${ }^{7-11}$

The aim of this paper is to prove the suitability of single-cell wholegenome amplification in combination with the Affymetrix $250 \mathrm{~K}$ SNP array to detect unbalanced translocations in single blastomeres. In addition, we investigated the feasibility of single-blastomere genotyping and its use as a confirmational method for the determination of

\footnotetext{
${ }^{1}$ Departments of Clinical Genetics, Maastricht University Medical Centre, Maastricht, The Netherlands; ${ }^{2}$ School for Oncology and Developmental biology (GROW), Maastricht University, Maastricht, The Netherlands; ${ }^{3}$ Department of Obstetrics \& Gynaecology (IVF laboratory), Maastricht University Medical Centre, Maastricht, The Netherlands *Correspondence: Dr SJC Stevens, Department of Clinical Genetics, Maastricht University Medical Centre, PO Box 5800 , 6202 AZ Maastricht, The Netherlands. Tel: +31 43 3871625; Fax: +31 43 3877901; E-mail: servi.stevens@mumc.nl

Received 17 August 2011; revised 19 January 2012; accepted 26 January 2012; published online 29 February 2012
} 
partial monosomies and of parental meiotic segregation of the translocation chromosomes.

\section{MATERIALS AND METHODS}

\section{Cell lines and clinical samples}

Epstein Barr virus-transformed lymphoblastoid cell lines (EBV-LCLs) with known CR were used for optimisation of the MDA-SNP technique (see Results). Blastomere samples $(n=146)$ were obtained from 36 embryos from 8 couples that were referred for PGD to our centre (Table 1). These embryos were previously diagnosed as chromosomally unbalanced by PGD singleblastomere FISH analysis. FISH results were scored as 'normal/balanced', 'unbalanced' or 'non-conclusive. Discarded embryos were dissociated in $\mathrm{Ca}^{2+}$ - and $\mathrm{Mg}^{2+}$-free Hank's balanced salt solution (HBSS) resulting in single and multiple blastomere samples. Samples were washed three times in HBSS and transferred to $2 \mu \mathrm{l} 1 \% \mathrm{PVP} / 1 \mathrm{x}$ PBS/phenol red. ${ }^{12}$ The last washing droplet served as a negative control. Blastomere samples were stored at $-20^{\circ} \mathrm{C}$ for at least $30 \mathrm{~min}$ before the WGA reaction.

National legislation with respect to PGD and PGS, in particular the extend of genetic embryo testing allowed, is the reason that only translocationassociated chromosomes could be analysed in this study. The study was approved by Institutional Medical Ethical Review Board and written informed consent was obtained from the participating couples. This approval comprehended only genetic analysis of the chromosomes involved in the translocation and therefore other chromosomes were not analysed.

\section{WGA by MDA}

Single cells were prepared for WGA as described by Spits et al. ${ }^{13}$ with minor modifications. Cells were lysed using $200 \mathrm{~mm} \mathrm{KOH} / 75 \mathrm{~mm} \beta$-mercaptoethanol. WGA was performed on the total lysate using the Repli-g Ultra-fast MDA kit (Qiagen, Düsseldorf, Germany) in a $20 \mu \mathrm{l}$ reaction mix at $30^{\circ} \mathrm{C}$ for $3 \mathrm{~h}$. The reaction was terminated at $65^{\circ} \mathrm{C}$ for $10 \mathrm{~min}$. MDA products were purified using the High Pure PCR Product Purification Kit (Roche Diagnostics, Mannheim, Germany). MDA products were quantified on a Nanodrop ND-1000 spectrophotometer (Thermo Fisher Scientific, Wilmington, NC, USA). DNA integrity was assessed by agarose gel electrophoresis. Samples yielding $\geq 1.5 \mu \mathrm{g}(30 \mathrm{ng} / \mu \mathrm{l})$ DNA were regarded as efficiently amplified. Genomic representation and putative contamination were assessed by STR marker analysis using the AmpFISTR Identifiler kit (Roche Applied Science, Mannheim, Germany). The assessment of the allelic drop-out (ADO) rate was based on heterozygous markers in this kit.

SNP array-based copy number and genotype analysis

Copy number $(\mathrm{CN})$ profiling was performed using the GeneChip Human Mapping $250 \mathrm{~K} \mathrm{~N}$ spI array (Affymetrix, Santa Clara, CA, USA) according to the manufacturer's instructions. SNP genotypes and SNP array-based CNs were determined using the Bayesian Robust Linear Model with Mahalanobis distance classifier (BRLMM) algorithm and the hidden Markov model (HMM)-based CN 4 (CN4) algorithm, ${ }^{14}$ implemented in the Genotyping Console software v3.0.2 (Affymetrix). In order to enhance signal to noise ratio and demarcation between regions of CN change, genomic smoothing (GS) length settings were adjusted as detailed in the Results. This setting is based on Gaussian distribution-based normalisation ${ }^{15-17}$ and increases signal to noise ratio in the $\mathrm{CN} 4$ calling algorithm before HMM-based $\mathrm{CN}$ segmentation. Software settings mentioned above were based on the manufacturer's white paper (BRLMM: an Improved Genotype Calling Method for the GeneChip Human Mapping $500 \mathrm{~K}$ Array Set, www.affymetrix.com 2006) and as explained previously. ${ }^{14-17}$

$\mathrm{CN}$ segments were defined as described previously, ${ }^{14,18}$ that is, a $\mathrm{CN}$ gain was called if $\geq 7$ consecutive SNPs showed increased signal and the concerning region was $\geq 200 \mathrm{Kbp}$ in size. A CN loss was called for $\geq 5$ consecutive SNPs with decreased signal and for regions $\geq 150 \mathrm{Kbp}$ in size. The reference data set consisted of 40 SNP arrays run on whole blood-derived DNA samples obtained from 40 normal $(46, \mathrm{XX})$ females, as suggested by the manufacturer. Karyotypes and array nomenclature were designated according to ISCN $2009^{19}$ and base pair positions were derived from the Genome Reference Consortium build GRCh37 (Ensembl release 56).

\section{RESULTS}

MDA amplification efficiency

MDA optimisation was done using a normal (46,XY) EBV-LCL. Amplification of single cells $(n=56)$ resulted in an average MDA yield of $4.3 \pm 1.8 \mu \mathrm{g}$ DNA, with MDA product length $>10 \mathrm{Kbp}$. Successful MDA (yield $>1.5 \mu \mathrm{g}$ ) was obtained in $93 \%$ of single LCLs (95\% confidence interval (CI): 83-97\%), with a combined amplification failure $(\mathrm{AF})$ and $\mathrm{ADO}$ rate of $18 \%$ (CI: $14-22 \%$; $\mathrm{AF}$ and $\mathrm{ADO}$ were based on 10 heterozygous markers). Blastomere samples $(n=124)$ showed a lower average yield $(3.49 \pm 1.0 \mu \mathrm{g}$ of DNA) compared with EBV-LCLs $(P<0.001)$ and single blastomeres gave a slightly lower yield compared with multiple blastomere samples, although this was not statistically significant (3.39 vs. $3.6 \mu \mathrm{g} ; P=0.058$ ) (Table 1). MDA-efficiency of blastomeres was $80 \%$ (CI: $71-87 \%)$ for single cells and 94\% (CI: $84-98 \%$ ) for multiple cell samples $(P=0.0278$, Fisher's exact test). ADO/AF rate was $25 \%$ (CI: $22-28 \%$ ) for single cells and $18 \%$ (CI: $15-22 \%$ ) for multi cell samples based on parental heterozygous markers detected by AmpFlSTR Identifiler kit. Negative control samples occasionally appeared to show MDA yield (ranging from $0.08-1.1 \mu \mathrm{g}$, mean $0.3 \mu \mathrm{g}$ ) but STR

Table 1 MDA results for single- and multi-cell blastomere samples

\begin{tabular}{|c|c|c|c|c|c|c|}
\hline \multirow[b]{2}{*}{ Case-cycle } & \multirow[b]{2}{*}{$\begin{array}{l}\text { Karyotype of the } \\
\text { translocation carrier }\end{array}$} & \multirow[b]{2}{*}{$\begin{array}{l}\text { Total number } \\
\text { of embryos }\end{array}$} & \multicolumn{2}{|c|}{ Successful amplification ${ }^{\text {a }}$} & \multicolumn{2}{|c|}{ Mean yield $(\mu g D N A)^{b}$} \\
\hline & & & $\begin{array}{l}\text { Single-cell } \\
\text { samples }\end{array}$ & $\begin{array}{l}\text { Multiple cell } \\
(>1) \text { samples }\end{array}$ & $\begin{array}{l}\text { Single-cell } \\
\text { samples }\end{array}$ & $\begin{array}{l}\text { Multiple cell } \\
(>1) \text { samples }\end{array}$ \\
\hline C1-1 & $45, X Y, \operatorname{der}(13 ; 14)(q 10 ; q 10)$ & 1 & $6 / 7$ & - & 3.1 & - \\
\hline C2-1 & $46, \mathrm{XY}, \mathrm{t}(5 ; 7)(\mathrm{q} 33.1 ; \mathrm{q} 22)$ & 4 & $9 / 10$ & $4 / 5$ & 3.6 & 3.8 \\
\hline C2-2 & $46, X Y, t(5 ; 7)(q 33.1 ; q 22)$ & 7 & $25 / 27$ & $7 / 7$ & 3.6 & 3.1 \\
\hline C2-3 & $46, X Y, t(5 ; 7)(q 33.1 ; q 22)$ & 3 & $18 / 22$ & $2 / 2$ & 3.2 & 3.4 \\
\hline C3-1 & $45, X X, \operatorname{der}(13 ; 14)(q 10 ; q 10)$ & 3 & $5 / 10$ & $3 / 4$ & 2.9 & 1.9 \\
\hline C4-1 & 45,XY,der(13;14)(q10;q10) & 1 & $0 / 3$ & $1 / 1$ & - & 3.6 \\
\hline C5-1 & 46,XY,t(1;9)(q32.3;q34.1) & 4 & $4 / 5$ & $8 / 8$ & 3.7 & 4.1 \\
\hline C6-1 & $46, \mathrm{XY}, \mathrm{t}(12 ; 19)(\mathrm{q} 24.1 ; \mathrm{p} 13.3)$ & 7 & $8 / 10$ & $14 / 15$ & 3.3 & 4.2 \\
\hline C7-1 & $46, X X, t(9 ; 19)(p 12 ; p 12)$ & 3 & - & 7/7 & - & 3.8 \\
\hline C8-1 & $45, X Y, \operatorname{der}(13 ; 14)(q 10 ; q 10)$ & 3 & - & $3 / 3$ & - & 2.4 \\
\hline Total & & 36 & $75 / 94$ & $49 / 52$ & 3.39 & 3.6 \\
\hline
\end{tabular}

a Single- vs multi-cell samples: $P=0.0278$.

bingle- vs multi-cell samples: $P=0.058$ 
marker analysis was negative in all cases, indicating amplification of DNA from non-human origin.

\section{Optimisation of single-cell CN profiling}

Single-cell SNP array analysis was optimised using EBV-LCLs derived from healthy donors and EBV-LCLs with the following known chromosomal aberrations: EBV-16310: 46,XX,der(3), $\mathrm{t}(3 ; 16)(\mathrm{p} 26.1 ; \mathrm{q} 23.1)$.arr 3p26.3p26.1(48,789-5,591,260)x1,16q23.1q24.3 (75,859,857-88,827,254)x3, EBV-18754: 46,XX,del(4)(q31.3).arr 4q31.23 $(148,332,457-150,492,322) x 3, \quad 4 q 31.3 q 35.2(153,757,121-191,167,888) x 1$ (the discrepancy between karyotype and SNP array findings can be explained by the fact that SNP array detected a small additional dup(4) (q31.23q31.23), which was not seen in light microscopy) and EBV18843: 46,XY,dup(X)(p22.2p22.2).arr Xp22.2(9,611,839-16,735,796)x2. Five single cells of each EBV-LCL were analysed in independent experiments using recommended default $\mathrm{CN}$ algorithm parameters in Genotyping Console (i.e., GS $=0.1 \mathrm{Mb}$, Transition Decay $=10 \mathrm{Mb}$, $\mathrm{HMM}$ prior value $=0.2$ for all $\mathrm{CN}$ states and $\mathrm{HMM} \mathrm{SD}=0.07$ for $\mathrm{CN}$ states of 0 (homozygous deletion), 1 (hemizygous deletion), 3 (gain of one allele) and 4 (gain of 2 alleles) and HMM SD $=0.09$ for $\mathrm{CN}$ state of 2 (normal diploid)). The expected chromosomal imbalances were detected in all single cells. However, the regions 1p, 16p, 19p, 19q and $22 q$ consistently showed large artefactual gains that were the likely effect of preferential amplification. The use of a SNP reference set based on single cells amplified by MDA corrected these artefactual gains (data not shown).

In addition to the known imbalance in EBV-LCLs, approximately 1500-2000 small, artefactual gains and losses were detected throughout the genome at the single-cell level, inherent to the single-cell MDA procedure. Therefore, the GS window setting was optimised for singlecell CN-calling applications. The GS filter uses Gaussian smoothing and increases signal to noise ratio in the $\mathrm{CN} 4$-calling algorithm before HMM-based CN segmentation. Single EBV-LCLs derived from a healthy donor were used for this. Increasing the GS led to a higher percentage of SNPs with $\mathrm{CN}=2$ (i.e., higher specificity), reaching a maximum level at approximately $90 \%$ (Figure 1a). Subsequently, the effect of increased GS on analytical resolution of the single-cell SNP array was assessed by analysing EBV-LCLs with known genomic aberrations. Using a GS value of $5 \mathrm{Mb}$ in the Genotyping Console software, a $5.5 \mathrm{Mb}$ monosomy in EBV-16310 (see above) could reproducibly be detected at the single-cell level. On the basis of these data and reasons explained in the discussion section, this GS value was chosen for subsequent analyses of surplus blastomeres (Figure $1 \mathrm{~b}$ ).

\section{SNP array-based CN analysis of human surplus blastomeres}

Following optimisation of the CN analysis settings, an MDA-SNP array study was initiated using blastomeres derived from discarded embryos with unbalanced translocations as detected by FISH (Table 1). The unbalanced genotype was detected at interphase FISH analysis of single blastomeres using a three-probe protocol for reciprocal translocations, with probes for both translocated segments and a probe for the centric segment (CS) of one of the chromosomes involved in the translocation. For Robertsonian translocations a two-probe strategy was used. Overall, 36 unbalanced embryos were available for analysis. Of these, 146 blastomere samples were derived, that is, 94 single and 52 multiple blastomeres samples, and amplified by MDA. In all, 75 single-cell samples (79.8\%) and 49 multiple blastomeres samples (94.2\%) could be analysed by SNP array as they showed successful amplification. Solely, the chromosomes involved in the translocation were analysed, as permission was obtained for a confirmative study only, and hence data from other chromosomes were not analysed (see Materials and Methods). Figure 2 shows an example of a single-blastomere $\mathrm{CN}$ analysis (Case 2-1, Emb 1.1, see also Table 2). At the genotype level, SNP array analysis showed identical signals for all individual blastomeres in 21/36 embryos available in this study, and for 11 of these SNP array confirmed the initial FISH signals. Out of 36 embryos, 15 were mosaic in SNP array signals (Table 2). SNP array analysis confirmed the FISH diagnosis in $31 / 36(86 \%)$ embryos, of which 8 were mosaic normal/unbalanced. In 5/36 (14\%) embryos, SNP array analysis showed $\mathrm{CN}=2$ for the translocation chromosomes, whereas the FISH diagnosis was 'unbalanced'.

\section{Combined SNP array-based $\mathrm{CN}$ analysis and genotyping to assess} translocation chromosome segregation

Next, the question was addressed whether SNP array-based genotype analysis of blastomeres could define the meiotic segregation pattern in a

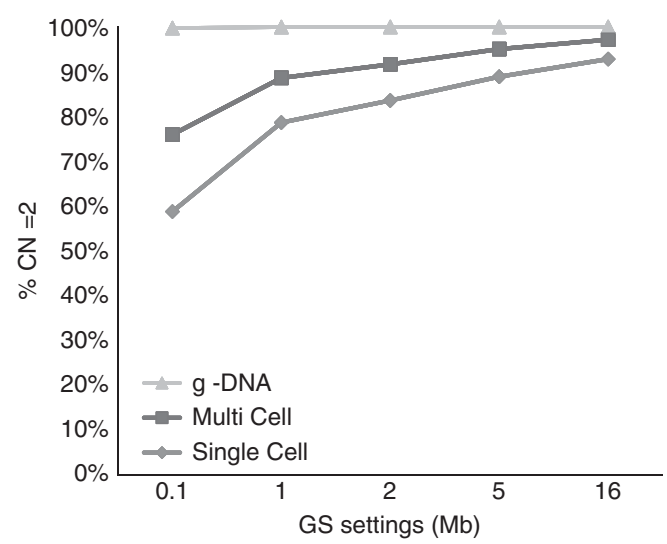

b

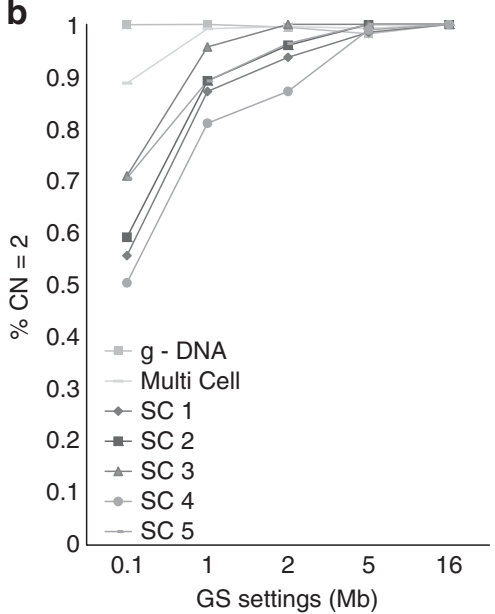

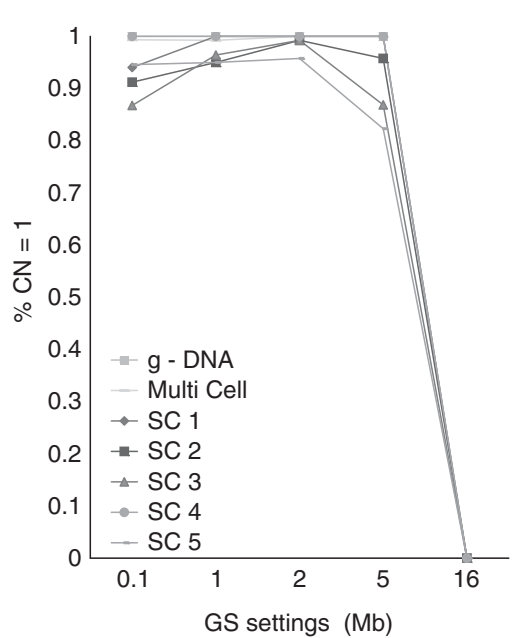

Figure 1 (a) Influence of GS settings on analytic specificity, that is, mean $\%$ of $\mathrm{CN}=2$ call (diploid) in normal EBV-LCLs. (single cells $n=14$, multi cells $n=10$, g-DNA samples $n=2$ ). The complete genome (represented by approximately 262000 SNP probes) was analysed. (b) Influence of GS settings on analytical resolution, that is, \% of correct $\mathrm{CN}$ calling from specific regions with known aberrations, that is, diploid region $3 p 26.1 \rightarrow 3 q$ tel $(C N=2)$ and partial monosomy 3p26.3 $\rightarrow$ 3p26.1 (CN=1) in EBV-LCL 16310 (single cells $n=5$, multi cells $n=1$, g-DNA sample $n=1$ ). 


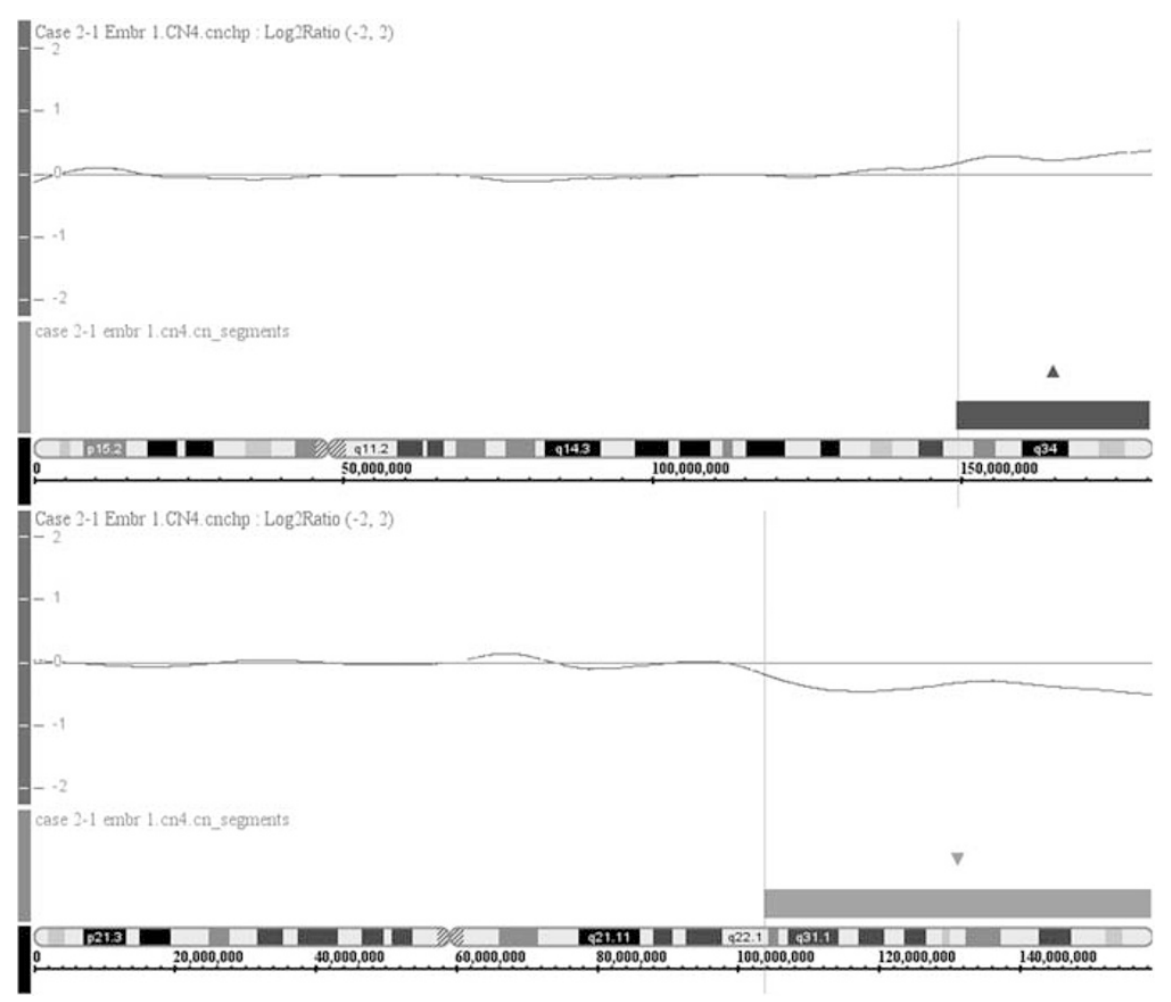

Figure 2 Single-cell CN analyses using the MDA-SNP array. An example of a single blastomere of case 2-1, embryo 1 is shown. Upper panel for both chromosomes shows the log2ratio of blastomere over reference signals (top: chromosome 5, bottom: chromosome 7). The lower panel for both chromosomes (top: chromosome 5, bottom: chromosome 7) shows the $\mathrm{CN}$ segments ('CN_segments') derived thereof (gains are indicated as blue blocks, losses are indicated as red blocks). The color reproduction of this figure is available at the European Journal of Human Genetics online.

a given translocation carrier. Informative SNP loci located in the translocation segments that appeared hemizygous by $\mathrm{CN}$ analysis were used for this, that is, those SNP loci for which the parents had opposing, homozygous calls (AA in the mother and $\mathrm{BB}$ in the father or vice versa). In disomic $(\mathrm{CN}=2)$ regions, opposing and homozygous SNP loci in the parents result in a heterozygous $(\mathrm{AB})$ call in the embryo. In hemizygous regions, these SNP loci revealed genetic contribution of one parental allele only (i.e., the allele of the partner of the translocation carrier), thereby confirming the partial monosomy detected by the $\mathrm{CN}$ analysis. A total of 80 hemizygous regions $(\mathrm{CN}=1)$ in 74 blastomeres ( 25 embryos) were analysed and in $67 / 80$ of these $(84 \%)$ only the partner of the translocation carrier contributed to the embryo's genotype, thereby confirming hemizygosity due to unbalanced translocation (see Figure 3 for an example). Disomic regions $(n=264)$ were confirmed by genotypic analysis in $90 \%$ of cases.

On the basis of parent-of-origin analysis and the $\mathrm{CN}$ calling, the meiotic segregation of the translocation chromosomes could be deduced. An example is given in Figure 3 (case 2-1, Emb 1), whereby the male translocation carrier contributed a chromosome 5 and a $\operatorname{der}(7) t(5 ; 7)$ (q33;q22) chromosome to the embryo due to adjacent-1 segregation in spermatogenesis. This resulted in a monosomy for the region $7 \mathrm{q} 22 \rightarrow 7 \mathrm{qter}$ (Figure 2 ), with only maternal contribution to the genotype for the region $7 \mathrm{q} 22 \rightarrow 7 \mathrm{qter}$ (Figure 3 ).

Finally, SNP array-based $\mathrm{CN}$ analysis of blastomeres from embryo 4 (C2-1) showed three normal/balanced blastomeres and two unbalanced blastomeres. However, SNP genotyping as explained above revealed only maternal contribution to the embryo's genotype.

FISH analyses could identify the putative meiotic segregation in the translocation carrier in 34/53 (64\%; CI: $50-77 \%)$ at the blastomere level, compared with 93/124 (75\%; CI: 66-82\%) in MDA-SNP array (not taking into account possible mitotic events).

\section{DISCUSSION}

FISH as a diagnostic method in PGD is rapidly being replaced by array-based methods. ${ }^{20}$ Both (BAC) array-CGH and SNP array have been used for detection of chromosomal imbalances in embryoderived samples. ${ }^{11,21-24,26}$ SNP arrays generate both qualitative (genotype) and quantitative $(\mathrm{CN})$ data, an advantage over array-CGH, which can only detect relative $\mathrm{CN}$ changes. ${ }^{20} \mathrm{SNP}$ array comprises $\mathrm{CN}$ profiling and genome-wide haplotyping, enabling detection of both chromosomal imbalances and monogenic disorders. ${ }^{25-27}$ Thus, SNP array holds the potential of providing a universal, 'off-the-shelf' PGD platform, applicable to a wide variety of genetic disorders.

We show the feasibility of detecting unbalanced translocations in single blastomeres derived from cleavage-stage embryos by MDA and subsequent SNP array analysis. MDA is an isothermal amplification method using Phi29 DNA polymerase and random priming. It has an extremely low error rate of 1 in $10^{6}-10^{7}$, gives high DNA yield and uniform WGA with relative low amplification bias or drop-out ${ }^{28-31}$ It yields DNA fragments sized $>10 \mathrm{Kbp}$, suitable for the Affymetrix array, which requires large DNA fragments for initial DNA digestion by restriction endonucleases. MDA efficiency and ADO rate were comparable to other studies ${ }^{32-35}$, and given the high SNP probe coverage $(>250,000)$, allowed for reliable CN profiling. The MDASNP array detection limit in this study was optimised to be $5 \mathrm{Mb}$, enabling detection of the vast majority of unbalanced segregation products for the vast majority of chromosome rearrangements. Only if all the unbalanced chromosome segments were $<5 \mathrm{MB}$, would a specific abnormal product not be undetected. From an interpretative 
Table 2 Results of SNP array-based CN calling on surplus blastomeres in comparison with diagnostic FISH results

\begin{tabular}{|c|c|c|c|c|c|c|c|c|}
\hline \multirow{3}{*}{$\begin{array}{l}\text { Case- } \\
\text { cycle }\end{array}$} & \multirow{3}{*}{$\begin{array}{l}\text { Embryo } \\
\text { blastomere }\end{array}$} & \multicolumn{4}{|c|}{ FISH analysis (no. of signals) } & \multirow[t]{2}{*}{ SNP analysis ( $C N$ state) } & \multicolumn{2}{|c|}{ SNP analysis } \\
\hline & & \multicolumn{2}{|c|}{ First chr } & \multicolumn{2}{|l|}{ Second chr } & & \multirow{2}{*}{$\begin{array}{c}\text { Confirmed } \\
\text { FISH genotype }\end{array}$} & \multirow{2}{*}{$\begin{array}{c}\text { Confirmed } \\
\text { FISH diagnosis }\end{array}$} \\
\hline & & CS & TS & CS & TS & First chr CS/first chr TS/second chr CS/second chr TS (no.) & & \\
\hline C1-1 & Emb 1.2 & 1 & & 0 & & & $0 / 6$ & \\
\hline \multirow[t]{2}{*}{ C2-1 } & Emb 1.1 & & 3 & 2 & 1 & 2/3/2/1 (4) & $4 / 4$ & $4 / 4$ \\
\hline & Emb 4.1 & & 2 & 1 & 1 & 2/2/2/2 (3); 1/1/3/3 (1); 3/3/3/3 (1) & $0 / 5$ & $2 / 5$ \\
\hline \multirow[t]{5}{*}{ C2-2 } & Emb 1.1 & & 1 & 2 & 3 & $2 / 1 / 2 / 3(2)$ & $2 / 2$ & $2 / 2$ \\
\hline & Emb 1.2 & & 0 & 2 & 1 & & $0 / 2$ & \\
\hline & Emb 2.1 & & 1 & 2 & 2 & $1 / 1 / 2 / 2(1) ; 2 / 1 / 2 / 3(1) ; 3 / 1 / 2 / 3(1) ; 2 / 1 / 1 / 3(1)$ & $1 / 4$ & $4 / 4$ \\
\hline & Emb 3.1 & & 1 & 1 & 2 & $2 / 2 / 2 / 2(5)$ & $0 / 5$ & $0 / 5$ \\
\hline & Emb 4.1 & & 1 & 2 & 3 & $2 / 1 / 2 / 3(1) ; 2 / 1 / 2 / 2(1) ; 1 / 2 / 2 / 2(1) ; 2 / 1 / 3 / 3(1) ; 2 / 2 / 2 / 2(1)$ & $1 / 5$ & $4 / 5$ \\
\hline \multirow[t]{5}{*}{ C2-3 } & Emb 1.1 & & 0 & 0 & 0 & $2 / 2 / 2 / 2(5)$ & $0 / 5$ & $0 / 5$ \\
\hline & Emb 1.2 & & 3 & 2 & 2 & & $0 / 5$ & \\
\hline & Emb 2.1 & & 2 & 1 & 0 & $2 / 3 / 2 / 1(6) ; 1 / 3 / 3 / 1(1)$ & $0 / 7$ & $7 / 7$ \\
\hline & Emb 3.1 & & 3 & 2 & 2 & 2/3/2/2 (1); $1 / 1 / 2 / 2(1) ; 3 / 2 / 2 / 2$ (1) 2/2/2/2 (5) & $1 / 8$ & $3 / 8$ \\
\hline & Emb 3.2 & & 2 & 2 & 2 & & $5 / 8$ & \\
\hline \multirow[t]{5}{*}{ C3-1 } & Emb 1.1 & 2 & & 1 & & $2 / c / 1 / c(4)$ & $4 / 4$ & $4 / 4$ \\
\hline & Emb 2.1 & 2 & & 1 & & $2 / c / 1 /{ }^{c}(2)$ & $2 / 2$ & $2 / 2$ \\
\hline & Emb 2.2 & 2 & & 0 & & & $0 / 2$ & \\
\hline & Emb 3.1 & 1 & & 2 & & $1 / \mathrm{C} / 2 /{ }^{\mathrm{C}}(2)$ & $2 / 2$ & $2 / 2$ \\
\hline & Emb 3.2 & 0 & & 0 & & & $0 / 2$ & \\
\hline C4-1 & Emb 1.1 & 1 & & 2 & & $1 /{ }^{c} / 2 /^{c}(1)$ & $1 / 1$ & $1 / 1$ \\
\hline \multirow{8}{*}{ C6-1 } & Emb 1.2 & 0 & 2 & & 2 & & $0 / 1$ & \\
\hline & Emb 2.1 & 1 & 2 & & 2 & $1 / 2 / 3 / 3(2)$ & $0 / 2$ & $2 / 2$ \\
\hline & Emb 3.1 & 4 & 3 & & 6 & $2 / 1 / 3 / 3(1)$ & $0 / 1$ & $1 / 1$ \\
\hline & Emb 4.1+2 & 2 & 1 & & 3 & 2/1/3/3 (8); 1/1/1/2 (1) & $8 / 9$ & $9 / 9$ \\
\hline & Emb 5.1 & $\mathrm{nc}$ & $\mathrm{nc}$ & & $\mathrm{nc}$ & $2 / 2 / 3 / 2(1)$ & $0 / 1$ & $1 / 1$ \\
\hline & Emb 5.2 & 2 & 1 & & 1 & & $0 / 1$ & \\
\hline & Emb 6.1+2 & 3 & 2 & & 2 & 3/2/1/2 (3); 3/3/1/2 (3) & $3 / 6$ & $6 / 6$ \\
\hline & Emb 7.1 frag. & $\mathrm{nc}$ & $\mathrm{nc}$ & & $\mathrm{nc}$ & $1 / 2 / 3 / 2(1) ; 2 / 2 / 2 / 2(1)$ & $0 / 2$ & $1 / 2$ \\
\hline \multirow[t]{4}{*}{ C7-1 } & Emb 1.1+2 & 1 & 1 & & 3 & $1 / 1 / 3 / 3(2)$ & $2 / 2$ & $2 / 2$ \\
\hline & Emb 2.1 & 2 & 3 & & 2 & $2 / 3 / 3 / 2(1) ; 2 / 3 / 3 / 3(1)$ & $1 / 2$ & $2 / 2$ \\
\hline & Emb 2.2 & 2 & 4 & & 2 & & $0 / 2$ & \\
\hline & Emb 3.1 & 2 & 2 & & 1 & $2 / 3 / 2 / 1(1) ; 2 / 1 / 3 / 2(1) ; 2 / 2 / 2 / 2(1)$ & $0 / 3$ & $2 / 3$ \\
\hline \multirow[t]{4}{*}{ C8-1 } & Emb 1.1 m.n.(7) & 0/2/0/0/2/0/0 & & 0/0/0/0/1/0/0 & & $1 / c / 1 / c(1)$ & $0 / 1$ & $1 / 1$ \\
\hline & Emb 2.1 m.n.(2) & $1 / 1$ & & $1 / 0$ & & $2 / c / 2 /{ }^{c}(1)$ & $0 / 1$ & $0 / 1$ \\
\hline & Emb 2.2 & 2 & & 2 & & & $1 / 1$ & \\
\hline & Emb 3.1 & 2 & & 5 & & $3 / c / 3 / c(1)$ & $0 / 1$ & $1 / 1$ \\
\hline
\end{tabular}

Abbreviations: CN, copy number; CS, centric segment; FISH, fluorescence in situ hybridisation; frag, fragmented DNA; m.n., multi nucleated; nc, non conclusive FISH analysis; SNP, single-nucleotide polymorphism; TS, translocated segment.

a'Confirmed FISH genotype' denotes those blastomeres in which MDA-SNP array-based CN analysis found the same CN as FISH (based on fluorescent signals).

b'Confirmed FISH diagnosis' denotes those embryos in which SNP array confirmed the FISH diagnosis, that is, 'normal/balanced', 'unbalanced' or 'non-conclusive'.

' In cases of Robertsonian translocations CN state is given for complete chromosomes (CS). Emb no. 1+2: both biopsied blastomeres had identical FISH results. 


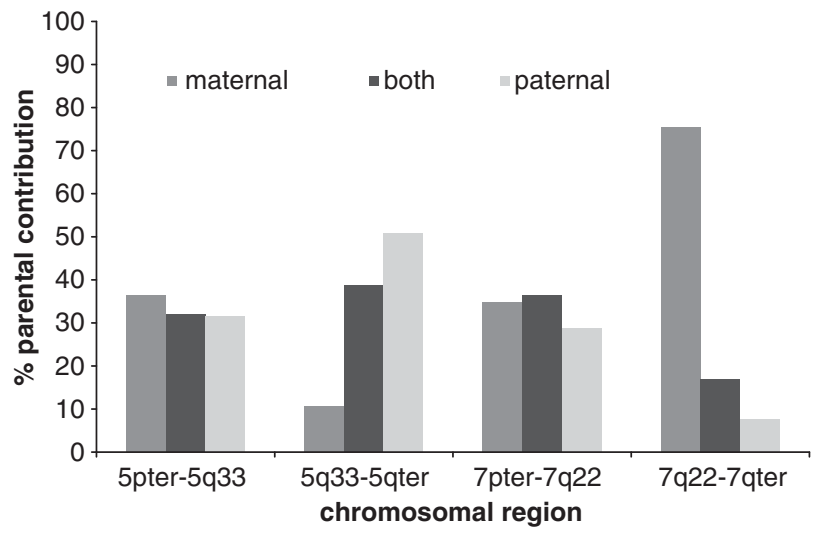

Figure 3 Genotype analysis showing parental contributions to the genotype of case $2-1$, embryo 1 , for the two centric segments (5pter $\rightarrow 5 q 33$ and $7 p t e r \rightarrow 7 q 22)$ and the two translocation segments $(5 q 33 \rightarrow 5 q$ ter and $7 q 22 \rightarrow 7 q$ ter). The analysis was based on informative parental loci, that is, opposing homozygous genotype calls in the couple. Blue bars indicate paternal-only contribution, without maternal contribution to the embryo's genotype and green bars indicate maternal-only contribution. Note that for the hemizygous region (7q22 $\rightarrow 7 q$ ter) associated with unbalanced translocation in the embryo, there is preferential genetic contribution of one parental allele only, that is, the allele of the partner of the translocation carrier (in this case maternal-only contribution). The color reproduction of this figure is available at the European Journal of Human Genetics online.

point-of-view, the $5 \mathrm{Mb}$ cut-off value ensures that small $\mathrm{CN}$ variants/ polymorphisms of unknown significance will not be scored.

Overall, the MDA-SNP array showed good correlation with the initial PGD FISH diagnosis. However, five surplus embryos (14\%) presented with a balanced/normal SNP genotype in all blastomeres but during initial PGD FISH had shown unbalanced blastomeres $(n=3)$ or a mosaic of unbalanced and balanced/normal blastomeres $(n=2)$. For these 5 embryos an average 3.8 blastomeres were tested, with all blastomeres giving identical results. Moreover, initial FISH failed for one of the two translocation chromosomes in 11 blastomeres $(20 \%)$, which is comparable to the proportion of single cell samples with failed MDA amplification (20\%) but higher than the proportion of multi cell samples with failed MDA amplification $(6 \%) .{ }^{35}$ MDA-SNP in our study was performed on blastomeres of surplus embryos, previously biopsied for FISH diagnosis. Blastomeres were included regardless of morphology or visibility of a nucleus, factors known to influence MDA efficiency. ${ }^{33}$ On the basis of the above, we anticipate that further sample optimisation will increase PGD efficiency with respect to the detection of chromosomal imbalances and hence, the number of embryos suitable for transfer, in comparison with FISH studies. ${ }^{36-39}$

There is some concern that SNP genotyping might detect predispositions to common and/or late-onset diseases. ${ }^{20}$ However, we do not think that today's SNP resolution is high enough, as only blocks of informative SNP loci can be reliably used for haplo- or genotype analysis. Although both array-CGH and SNP array are able to detect male triploid embryos (based on the X-chromosome: pseudoautosomal region ratio), female triploidies can only be detected by the latter. Although not shown in this study, SNP array-derived genotypes will mark triploidies based on the analysis of informative loci obtained from the parental haplotypes. ${ }^{27}$ SNP genotyping will also identify presumed haploid blastomeres, as shown here for embryo 4 (C2-1). This embryo probably derived from a parthenogenetically activated oocyte (1PN) as genotyping showed only maternal contribution to the embryo's genotype with no evidence of a paternal genome being present. Such embryos are non-viable and thus unsuitable for transfer but remain undetected by $\mathrm{CN}$ analysis alone, because there is no relative change in $\mathrm{CN}$.

The main advantage of array-CGH is that it is currently much faster than SNP array, ${ }^{9,21-24}$ enabling a diagnosis within 1.5 days without the need for embryo freezing. The present MDA-SNP array protocol requires 4 days. A possible future scenario would be trophectoderm (TE) biopsy at day 5 with subsequent vitrification of blastocysts. ${ }^{40}$ This would allow for embryo accumulation from multiple stimulation cycles and embryo transfer in an unstimulated cycle, which, from a biological point of view, might be beneficial.

Next to array-based methods, microsatellite marker-based methods have recently been described to detect translocations in embryos. ${ }^{41,42}$ These methods show improved identification of meiotic chromosome segregation compared with FISH protocol, and combine laboratory flow for structural chromosome analysis and monogenic testing. However, unlike SNP array, they still require a custom pre-PGD work-up, tailored to the couple's specific translocation.

PGD efficiency may be improved not only by optimizing the diagnostic technique used, but also by clinical specimen type and by aneuploidy screening. The optimal clinical specimen type for PGD, that is, polar bodies, cleavage-stage embryo-derived blastomeres or blastocyst-derived TE cells, has not yet been defined. ${ }^{43}$ It is crucial in this matter to differentiate between the aim of PGD, which is to provide a couple with an unaffected child and the aim of PGS, where screening for euploid embryos is thought to increase pregnancy rate. ${ }^{20}$

Polar bodies have the advantage that only meiotic errors are detected and would therefore be the ideal specimen for PGS. Prospective, randomized-controlled trials, currently ongoing, ${ }^{44}$ will need to elucidate this. In PGD, a polar body approach would be applicable only to female carriers of a genetic condition. Although many cleavage stage embryos are chromosomally mosaic, ${ }^{45-47}$ they may still be used for PGD, as long as the procedure is performed for known genetic aberrations only, without additional PGS. ${ }^{48,49}$ Some studies claim that the problem of mosaicism can be overcome by using TE cells for diagnosis, ${ }^{50-52}$ but a high mosaicism level has been reported for blastocyst-stage embryos as well, albeit at a lower rate per blastocyst. ${ }^{53}$ It is currently unknown whether TE will be better suited for PGD or PGS than cleavage-stage blastomeres as to the best of our knowledge, no randomized controlled trial has been performed to compare diagnostic outcome of both cell types.

In conclusion, we demonstrated the feasibility of SNP array-based detection of unbalanced translocations in single human blastomeres as well as parental origin of monosomies. Pilot data show improved diagnostic performance of the MDA-SNP technique compared with single blastomere FISH. Our results justify further studies to determine the putative benefits (improved take-a-baby-home rate) of additional genome-wide aneuploidy screening in blastomeres and/or TE cells from embryos of translocation carriers referred for PGD.

\section{CONFLICT OF INTEREST}

The authors declare no conflict of interest.

\section{ACKNOWLEDGEMENTS}

Epstein Barr virus-transformed lymphoblastoid cell lines (EBV-LCLs) were kindly provided by Dr Dominique Smeets, University Medical Centre Nijmegen, The Netherlands and by Prof Dr Jaap M Middeldorp and co-workers, VU Medical Centre, Amsterdam, The Netherlands. We thank all members of the IVF laboratory Maastricht University Medical Centre, The Netherlands for providing blastomeres samples used in this study. 
1 Scriven PN, Handyside AH, Ogilvie CM: Chromosome translocations: segregation modes and strategies for preimplantation genetic diagnosis. Prenat Diagn 1998; 18: 1437-1449.

2 Fryns J-P, Van Buggenhout G: Structural chromosome rearrangements in couples with recurrent fetal wastage. Eur J Obstet Gynecol Reprod Biol 1998; 81: 171-176.

3 Geraedts JPM, De Wert GMWR: Preimplantation genetic diagnosis. Clin Genet 2009; 76: 315-325.

4 Coonen E, Hopman AH, Geraedts JP, Ramaekers FC: Application of in-situ hybridization techniques to study human preimplantation embryos: a review. Hum Reprod Update 1998; 4: 135-152.

5 Wells D: Advances in preimplantation genetic diagnosis. Eur J Obstet Gynecol Reprod Biol 2004; 115 (Suppl. 1): S97-S101.

6 Wilton L: Preimplantation genetic diagnosis and chromosome analysis of blastomeres using comparative genome hybridization. Hum Reprod Update 2005; 11: 33-41.

7 Hellani A, Abu-Amero K, Azouri J, El-Akoum S: Successful pregnancies after application of array-comparative genomic hybridization in PGS-aneuploidy screening. Reprod Biomed Online 2008; 17: 841-847.

8 Harper JC, Harton G: The use of arrays in preimplantation genetic diagnosis and screening. Fertil Steril 2010; 94: 1173-1177.

9 Gutiérrez-Mateo C, Colls P, Sánchez-García J et al: Validation of microarray comparative genomic hybridization for comprehensive chromosome analysis of embryos. Fertil Steril 2011; 95: 953-958.

10 Treff NR, Su J, Tao X, Levy B, Scott Jr RT: Accurate single cell 24 chromosome aneuploidy screening using whole genome amplification and single nucleotide polymorphism microarrays. Fertil Steril 2010; 94: 2017-2021.

11 Treff NR, Northrop LE, Kasabwala K, Su J, Levy B, Scott Jr RT: Single nucleotide polymorphism microarray-based concurrent screening of 24-chromosome aneuploidy and unbalanced translocations in preimplantation human embryos. Fertil Steril 2011; 95: 1606-1612.

12 Dreesen J, Drüsedau M, Smeets $\mathrm{H}$ et al: Validation of preimplantation genetic diagnosis by PCR analysis: genotype comparison of the blastomere and corresponding embryo, implications for clinical practice. Mol Hum Reprod 2008; 14 573-579.

13 Spits C, Le Caignec C, De Rycke $M$ et al: Whole-genome multiple displacement amplification from single cells. Nat Protoc 2006; 1: 1965-1970.

14 Hehir-Kwa JY, Egmont-Petersen M, Janssen IM, Smeets D, van Kessel AG, Veltman JA: Genome-wide copy number profiling on high-density bacterial artificial chromosomes, single-nucleotide polymorphisms, and oligonucleotide microarrays: a platform comparison based on statistical power analysis. DNA Res 2007; 14: 1-11.

15 Di X, Matsuzaki H, Webster TA et al: Dynamic model based algorithms for screening and genotyping over $100 \mathrm{~K}$ SNPs on oligonucleotide microarrays. Bioinformatics 2005; 21: 1958-1963.

16 Bolstad BM, Irizarry RA, Astrand M, Speed TP: A Comparison of normalization methods for high density oligonucleotide array data based on bias and variance. Bioinformatics 2003; 19: 185-193.

17 Nannya Y, Sanada M, Nakazaki K et al: A Robust algorithm for copy number detection using high-density oligonucleotide single nucleotide polymorphism genotyping arrays. Cancer Res 2005; 65: 6071-6079.

18 Vermeesch JR, Fiegler $\mathrm{H}$, de Leeuw $\mathrm{N}$ et al: Guidelines for molecular karyotyping in constitutional genetic diagnosis. Eur J Hum Genet 2007; 15: 1105-1114.

19 Shaffer LG, Slovak ML, Campbell LJ (eds): ISCN(2009) An International System for Human Cytogenetic Nomenclature. Basel: S Karger, 2009.

20 Harper JC, Sengupta SB: Preimplantation genetic diagnosis: State of the ART 2011. Hum Genet 2012; 131: 175-186.

21 Montag M, Köster K, van der Ven K, Bohlen U, Bender F, van der Ven H: Kombinierte Translokations- und Aneuploidieuntersuchungen nach Polkörperbiopsie und arrayComparative Genomic Hybridisation. J Reproduktionsmed Endokrinol 2010; 7: 498-502.

22 Alfarawati S, Fragouli E, Colls P, Wells D: First births after preimplantation genetic diagnosis of structural chromosome abnormalities using comparative genomic hybridization and microarray analysis Hum. Reprod Advance Access 2011; 26: 1560-1574.

23 Fiorentino F, Spizzichino L, Bono S et al: PGD for reciprocal and Robertsonian translocations using array comparative genomic hybridization. Hum Reprod 2011; 26: 1925-1935.

24 Rius M, Obradors A, Daina G et al: Detection of unbalanced chromosome segregations in preimplantation genetic diagnosis of translocations by short comparative genomic hibridization. Fertil Steril 2011; 96: 134-142.

25 Treff NR, Tao X, Schillings WJ, Bergh PA, Scott Jr RT, Levy B: Use of single nucleotide polymorphism microarrays to distinguish between balanced and normal chromosomes in embryos from a translocation carrier. Fertil Steril 2011; 96: e58-e65.

26 Vanneste E, Melotte C, Voet T et al: PGD for a complex chromosomal rearrangement by array comparative genomic hybridization. Hum Reprod 2011; 26: 941-949.
27 Handyside AH, Harton GL, Mariani B et al: Karyomapping: a universal method for genome wide analysis of genetic disease based on mapping crossovers between parental haplotypes. J Med Genet 2010; 47: 651-658.

28 Dean FB, Hosono S, Fang $L$ et al: Comprehensive human genome amplification using multiple displacement amplification. Proc Natl Acad Sci USA 2002; 99: 5261-5266.

29 Coskun S, Alsmadi O: Whole genome amplification from a single cell: a new era for preimplantation genetic diagnosis. Prenat Diagn 2007; 27: 297-302.

30 Woyke T, Sczyrba A, Lee J et al: Decontamination of MDA reagents for single cell whole genome amplification. PLoS One 2011; 6: e26161.

31 Le Caignec C, Spits C, Sermon K et al: Single-cell chromosomal imbalances detection by array CGH. Nucleic Acids Res 2006; 34: e68.

32 Harton GL, De Rycke M, Fiorentino F et al: ESHRE PGD consortsium best practice guidelines for amplification-based PGD. Hum Rep 2011; 26: 33-40.

33 Spits C, Sermon K: PGD for monogenic disorders:aspects of molecular biology. Prenat Diagn 2009; 29: 50-56.

34 Renwick PJ, Trussler J, Ostad-Saffari E et al: Proof of principle and first cases using preimplantation genetic haplotyping-a paradigm shift for embryo diagnosis. Reprod Biomed Online 2006; 13: 110-119.

35 Ling J, Zhuang G, Tazon-Vega B et al: Evaluation of genome coverage and fidelity of multiple displacement amplification from single cells by SNP array. Mol Hum Reprod 2009; 15: 739-747.

36 Treff NR, Levy B, Su J, Northrop LE, Tao X, Scott Jr RT: SNP microarray-based 24 chromosome aneuploidy screening is significantly more consistent than FISH. Mol Hum Reprod 2010; 16: 583-589.

37 Munné S, Sandalinas M, Escudero T, Fung J, Gianaroli L, Cohen J: Outcome of preimplantation genetic diagnosis of translocations. Fertil Steril 2000; 73 : 1209-1218.

38 Velilla E, Escudero T, Munné S: Blastomere fixation techniques and risk of misdiagnosis for preimplantation genetic diagnosis of aneuploidy. Reprod Biomed Online 2002; 4: 210-217.

39 Li M, DeUgarte CM, Surrey M, Danzer H, DeCherney A, Hill DL: Fluorescence in situ hybridization reanalysis of day- 6 human blastocysts diagnosed with aneuploidy on day 3. Fertil Steril 2005; 84: 1395-1400.

40 Van Landuyt L, Stoop D, Verheyen $\mathrm{G}$ et al: Outcome of closed blastocyst vitrification in relation to blastocyst quality: evaluation of 759 warming cycles in a single-embryo transfer policy. Hum Reprod 2011; 26: 527-534.

41 Traversa MV, Carey L, Leigh D: A molecular strategy for routine preimplantation genetic diagnosis in both reciprocal and Robertsonian translocation carriers. Mol Hum Reprod 2010; 16: 329-337.

42 Fiorentino F, Kokkali G, Biricik A et al: Polymerase chain reaction-based detection of chromosomal imbalances on embryos: the evolution of preimplantation genetic diagnosis for chromosomal translocations. Fertil Steril 2010; 94: 2001-2011.

43 Simpson JL: Preimplantation genetic diagnosis at 20 years. Prenat Diagn 2010; 30: 682-695.

44 Geraedts J, Montag M, Magli MC et al: Polar body array CGH for prediction of the status of the corresponding oocyte. Part I: clinical results. Hum Reprod 2011; 26: 3173-3180.

45 Vanneste $\mathrm{E}$, Voet $\mathrm{T}$, Le Caignec $\mathrm{C}$ et al: Chromosome instability is common in human cleavage-stage embryos. Nat Med 2009; 15: 577-583.

46 Harper JC, Coonen E, Handyside AH, Winston RM, Hopman AH, Delhanty JD: Mosaicism of autosomes and sex chromosomes in morphologically normal, monospermic preimplantation human embryos. Prenat Diagn 1995; 15: 41-49.

47 Johnson DS, Gemelos G, Baner J et al: Preclinical validation of a microarray method for full molecular karyotyping of blastomeres in a 24-h protocol. Hum Reprod 2010; 25: 1066-1075.

48 Mastenbroek S, Twisk M, van Echten-Arends J et al: In vitro fertilization with preimplantation genetic screening. N Engl J Med 2007; 357: 9-17.

49 Mastenbroek S, Twisk M, van der Veen F, Repping S: Preimplantation genetic screening: a systematic review and meta-analysis of RCTs. Hum Reprod Update 2011; 17: 454-466.

50 Northrop LE, Treff NR, Levy B, Scott Jr RT: SNP microarray-based 24 chromosome aneuploidy screening demonstrates that cleavage-stage FISH poorly predicts aneuploidy in embryos that develop to morphologically normal blastocysts. Mol Hum Reprod 2010; 16: 590-600.

51 Schoolcraft WB, Fragouli E, Stevens J, Munne S, Katz-Jaffe MG, Wells D: Clinical application of comprehensive chromosomal screening at the blastocyst stage. Fertil Steril 2010; 94: 1700-1706.

52 Fragouli E, Lenzi M, Ross R, Katz-Jaffe M, Schoolcraft WB, Wells D: Comprehensive molecular cytogenetic analysis of the human blastocyst stage. Hum Reprod 2008; 23 : 2596-2608.

53 van Echten-Arends J, Mastenbroek S, Sikkema-Raddatz B et al: Chromosomal mosaicism in human preimplantation embryos: a systematic review. Hum Reprod Update 2011; 17: 620-627. 